\title{
Análise das Ações Preventivas de Úlceras por Pressão por Meio da Escala de Braden
}

\author{
Analysis of Preventive Actions of Pressure Ulcers through the Braden Scale
}

\author{
Análisis de las Acciones de Prevención de las Úlceras por Presión a través \\ de la Escala de Braden
}

\author{
Belarmino Santos de Sousa Júnior ${ }^{1}$, Cintia de Carvalho Silva², Fernando Hiago da Silva Duarte ${ }^{3}$, \\ Ana Elza Oliveira de Mendonça ${ }^{4}$, Daniele Vieira Dantas ${ }^{5}$
}

\begin{abstract}
RESUMO
Objetivo: Analisar as medidas preventivas para o desenvolvimento de úlceras por pressão em pacientes admitidos em uma Unidade de Terapia Intensiva de um hospital da rede privada no município de Caruaru, no estado de Pernambuco, adotadas pela equipe de Enfermagem a partir dos escores da Escala de Braden. Métodos: Estudo descritivo, com abordagem quantitativa; a população constituiu-se de pacientes acamados, de ambos os sexos, com escore $\leq 16$ na Escala de Braden. Para a coleta de dados, utilizaram-se um instrumento estruturado e uma análise dos prontuários, após aprovação do Comitê de Ética em Pesquisa. Resultados: Participaram do estudo 20 pacientes. Identificaram-se deficiências na Sistematização da Assistência de Enfermagem, bem como na aplicabilidade de medidas preventivas, o que revelou o surgimento de úlceras por pressão em 95\% dos pesquisados. Conclusão: Observou-se um déficit de ações da Enfermagem voltadas à prevenção de úlceras por pressão, evidenciadas na relação causal entre as variáveis e, consequentemente, no surgimento das úlceras, contribuindo, assim, para demonstrar a importância da adoção de medidas preventivas por meio da Escala de Braden e da Sistematização da Assistência de Enfermagem.
\end{abstract}

DESCRITORES: Unidade de Terapia Intensiva. Úlcera por pressão. Cuidados de Enfermagem.

\begin{abstract}
Objective: To analyze the preventive measures for the development of pressure ulcers in patients admitted at an Intensive Care Unit of a private hospital from Caruaru, Pernambuco, Brazil, which were adopted by the Nursing team based on Braden Scale scores. Methods: This is a descriptive study, with a quantitative approach; the population consisted of bedridden patients, of both sexes, and a score $\leq 16$ on Braden Scale. For data collection, we used a structured instrument and an analysis of medical records, after approval by the Research Ethics Committee. Results: The study included 20 patients. Deficiencies were identified in the Systematization of Nursing Assistance, as well as on the applicability of preventive measures, which showed the appearance of pressure ulcers in 95\% of the respondents. Conclusion: There was a lack of Nursing actions aimed at preventing pressure ulcers, which were highlighted in the causal relation between the variables and, consequently, in the appearance of ulcers, thus helping to demonstrate the importance of adopting preventive measures through Braden Scale and Systematization of Nursing Assistance.
\end{abstract}

DESCRIPTORS: Intensive Care Units. Pressure ulcer. Nursing care.

\footnotetext{
Trabalho realizado na Unidade de Terapia Intensiva de um hospital privado - Caruaru (PE), Brasil.

'Discente em Enfermagem pela Universidade Potiguar (UnP) - Natal (RN), Brasil. Endereço para correspondência: Rua Barão de Lucena, 62, torre 2 apto. 1.703 - CEP: 59066-285 - Natal (RN), Brasil - E-mail: sousajunyor@gmail.com

${ }^{2}$ Mestre em Enfermagem pela Universidade de Pernambuco (UPE). Professora do curso de bacharelado em Enfermagem da Faculdade ASCES - Caruaru (PE), Brasil. ${ }^{3}$ Especialista em Unidade de Terapia Intensiva pela Faculdade Metropolitana de Ciências e Tecnologia (FAMEC). Mestrando em Enfermagem pela Universidade Federal do Rio Grande do Norte (UFRN) - Natal (RN), Brasil.

${ }^{4}$ Especialista em Unidade de Terapia Intensiva pela Faculdade São Camilo do Rio de Janeiro (FELM) e em Nefrologia pela Universidade Federal de São Paulo (UNIFESP). Doutora em Ciências da Saúde pela UFRN - Natal (RN), Brasil.

${ }^{5}$ Doutora em Ciências da Saúde pela UFRN. Professora Adjunta do Departamento de Enfermagem da UFRN - Natal (RN), Brasil.

Fonte de financiamento: O primeiro autor é bolsista do Programa Institucional de Bolsas de Iniciação Científica (PIBIC) do Conselho Nacional de Desenvolvimento Científico e Tecnológico (CNPQ).

Artigo recebido em: 17/09/2015 - Aceito para publicação em: 11/12/2015
} 


\section{RESUMEN}

Objetivo: Analizar las medidas de prevención para el desarrollo de las úlceras por presión en pacientes ingresados en Unidad de Cuidados Intensivos, en un hospital de la rede privada de Caruaru, Pernambuco, Brasil, adoptadas por el equipo de Enfermería desde las puntuaciones de la Escala de Braden. Métodos: Estudio descriptivo, con enfoque cuantitativo; la población estuvo conformada por pacientes postrados en cama, de ambos sexos, y con una puntuación s16 en la Escala de Braden. Para la recolección de datos, fueron utilizados un instrumento estructurado y el análisis de la historia clínica, tras su aprobación por el Comité de Ética de la Investigación. Resultados: Se incluyeron 20 pacientes. Fueron identificadas deficiencias en la Sistematización de la Asistencia de Enfermería, y en la aplicabilidad de las medidas preventivas, lo que muestran la aparición de úlceras por presión en un 95\% de los encuestados. Conclusión: Se observó un déficit de acciones de la Enfermería dirigidas a la prevención de úlceras por presión, evidenciadas en la relación de causalidad entre las variables y, en consecuencia, la aparición de úlceras, lo que ayuda a demostrar la importancia de adoptar medidas preventivas a través de la Escala de Braden y SAE.

DESCRIPTORES: Unidades de Cuidados Intensivos. Úlcera por presión. Atención de enfermería.

\section{INTRODUÇÃO}

O surgimento de úlceras por pressão (UP) se mantém elevado, apesar dos avanços tecnológico e científico na área de saúde. De acordo com um estudo brasileiro, a sua incidência varia de 23,1 a 59,5\%, principalmente em pacientes internados em Unidades de Terapia Intensiva $(\mathrm{UTI})^{1}$. Neste cenário típico para o surgimento de lesões, as UP se constituem em um dos principais problemas de pele que acometem pacientes críticos durante internações hospitalares ${ }^{2}$.

Nesse sentido, o surgimento de UP durante a internação hospitalar implica em um indicador relevante da qualidade prestada, como consta na Portaria MS/GM 529/2013, que é o Programa Nacional de Segurança do Paciente (PNSP). Este tem como principal objetivo monitorar a incidência de UP, além de outros programas que visem minimizar esses e outros agravos ${ }^{3}$.

O desenvolvimento da UP ocorre quando a área afetada sofre morte celular, portanto o tecido mole é comprimido entre uma proeminência óssea e uma superfície rígida durante um período prolongado ${ }^{4}$. Pesquisadores na área afirmam que, atualmente, existem vários termos para defini-la: escara, úlcera de decúbito, úlcera de compressão ou ferida de pressão. No entanto, o termo "úlcera por pressão" é o que se encontra mais apropriado, na medida em que se constata ser a pressão exercida sobre os tecidos e as proeminências ósseas, em relação ao leito, o fator etiológico mais importante para essas lesões ${ }^{5}$.

A etiologia para o surgimento das UP acontece a partir de dois determinantes etiológicos críticos, como a intensidade e a duração da pressão, agregados à tolerância dos tecidos para suportarem determinada pressão, principalmente em proeminências ósseas ${ }^{6}$. Tais lesões estão relacionadas a diversos fatores. No que concerne aos intrínsecos ao paciente, podem-se destacar a idade, o estado nutricional, a imobilidade, as anemias, as infecções, a sensibilidade cutânea e as incontinências. Referindo-se aos fatores extrínsecos, decorrentes do ambiente, os mais importantes são: higiene deficiente, umidade, cisalhamento do lençol, ausência de mudança de decúbito, uso de instrumentos ortopédicos, sondas fixadas de forma inadequada, uso incorreto de agentes físicos/químicos, tipos de colchões e assentos inadequados ${ }^{1}$.

A amplitude das consequências do desenvolvimento dessas lesões, seja em termos econômicos ou de sofrimento humano, despertou a atenção não só de estudiosos, pesquisadores ou profissionais, mas também de órgãos governamentais ligados à saúde, de modo que, principalmente, a prevenção das UP tem tido prioridade no desenvolvimento de políticas que orientam a prática fundamentada em bases científicas ${ }^{7}$.

Assim, com a finalidade de melhorias na atenção aos pacientes em risco para o desenvolvimento de UP e, consequentemente, de reduzir a sua ocorrência, alguns serviços de saúde elaboram protocolos, os quais contam com medidas preventivas a serem adotadas conforme o grau de risco do paciente, avaliado por escalas padronizadas, sendo a de Braden a mais utilizada ${ }^{8}$.

A Escala de Braden (EB) foi criada por Braden e Bergstron, com o objetivo de diminuir a incidência de UP no serviço onde trabalhavam ${ }^{9}$. Ela foi traduzida e adaptada para a língua portuguesa por Wana Paranhos em 
1999, permitindo adequada utilização no Brasil. A EB está amparada na fisiopatologia das UP e permite uma avaliação dos aspectos relevantes ao desenvolvimento da úlcera, segundo seis parâmetros: percepção sensorial, umidade, mobilidade e atividade, nutrição, fricção e cisalhamento. Os cinco primeiros subescores recebem uma pontuação que varia de um a quatro, enquanto que fricção e cisalhamento, de um a três. A soma de cada subescore resulta na estratificação em faixas, em que os menores valores indicam as piores condições ${ }^{10}$.

Neste contexto, a identificação dos fatores individuais de risco é útil para direcionar a sistematização do cuidado e a terapêutica. A EB é um instrumento de sistematização que sinaliza ao profissional enfermeiro uma das etapas da Sistematização da Assistência de Enfermagem (SAE), identificando o risco que o paciente tem em desenvolver a UP $^{11}$. A implementação da SAE é de extrema relevância para a prevenção de UP, oferecendo ao paciente aquilo que é a essência da profissão, ou seja, cuidar do outro quando este não consegue fazê-lo ou precisa de auxílio, como orientação ou supervisão. A SAE possibilita uma constante reflexão sobre a escolha das intervenções visando à prevenção de UP, fazendo com que o enfermeiro desenvolva a sua capacidade para tomar as melhores decisões com crescente autonomia e cientificidade ${ }^{12}$.

Desse modo, a viabilização dos subsídios para a SAE advém da Taxonomia II da North American Nursing Diagnosis Association (NANDA I), que apresenta os diagnósticos de Enfermagem e das ligações NANDA I - NIC, com as intervenções de Enfermagem, fortalecendo ainda mais a cientificidade da profissão ${ }^{13}$.

Cabe ressaltar que existem diversas maneiras de sistematizar a assistência de Enfermagem, entre as quais estão os planos de cuidados, os protocolos, a padronização de procedimentos e o processo de Enfermagem. São diferentes formas de desenvolver o cuidado, ou seja, diversos métodos podem ser empregados para solucionar uma situação real em um determinado tempo, a fim de alcançar resultados positivos para a saúde dos pacientes ${ }^{12}$. Sendo assim, a SAE vem para somar e aderir o planejamento, a execução, o controle e a avaliação das ações de cuidados direto e indireto aos pacientes ${ }^{11}$.

Diante da importância do tema, objetivou-se analisar as medidas preventivas para o desenvolvimento de UP, em pacientes admitidos em uma UTI, adotadas pela equipe de Enfermagem, baseando-se na EB.

\section{MÉTODOS}

Trata-se de um estudo descritivo, com abordagem quantitativa, relacionado à assistência de Enfermagem no âmbito de prevenção das UP aos pacientes internados na em UTI.

O estudo foi realizado na UTI de um hospital da rede privada no município de Caruaru, em Pernambuco, de janeiro a maio de 2014. A população foi constituída por pacientes acamados, considerados críticos, de ambos os sexos, internados na unidade da referida instituição, considerando a quantidade de leitos. A amostra constou de 20 sujeitos, selecionados intencionalmente, com base nos seguintes critérios de inclusão: ter mais de 18 anos; escore na escala de Braden $\leq 16$; não apresentar UP no momento de admissão na UTI; permanecer internado na UTI por, no mínimo, 72 horas. Os critérios de exclusão foram: ser transferido para outra unidade hospitalar; obter alta da UTI e paciente evoluir a óbito.

O Termo de Consentimento Livre e Esclarecido foi lido e assinado por todos os pesquisados e/ou seus responsáveis, no qual assegurou-lhes o sigilo dos dados e o anonimato. O projeto de pesquisa foi apreciado e aprovado pelo Comitê de Ética e Pesquisa, sob número 22350513.6.0000.5203, em observância à Resolução 466/12 do Conselho Nacional de Saúde.

Para este estudo, foram utilizados como fonte de dados os prontuários dos pacientes selecionados, a fim de coletar informações inerentes à hipótese diagnóstica, tais como: idade, resultados de exames laboratoriais, registro da evolução, avaliação nutricional, presença de comorbidades e prescrições medicas e de Enfermagem.

$\mathrm{Na}$ primeira etapa da pesquisa, realizou-se a classificação do paciente pela EB para avaliar o risco de desenvolver UP. Tal instrumento é composto de seis subescalas: percepção sensorial, umidade, atividade, mobilidade, nutrição, fricção e cisalhamento. Das seis subescalas, três medem determinantes clínicos de exposição para intensa e prolongada pressão (percepção sensorial, atividade e mobilidade) e três mensuram a tolerância da pele para a pressão (umidade, nutrição, fricção e cisalhamento). As cinco primeiras são pontuadas com um escore que varia de um (menos propício) a quatro (mais propício); a sexta, referente à fricção e ao cisalhamento, é classificada com escore de um a três. Cada subescala é acompanhada de um título, e cada nível de um conceito de uma ou duas frases descrevendo ou qualificando as condições a serem avaliadas. $\mathrm{O}$ escore total na $\mathrm{EB}$ varia de 6 a 23. 
Portanto, o risco para o desenvolvimento de UP dependerá do escore da $\mathrm{EB}$, verificado por meio da avaliação do paciente, descrito da seguinte forma: sem risco - 19 a 23 pontos; baixo - 15 a 18 pontos; moderado - 13 a 14 pontos; alto -10 a 12 pontos; $\leq 9$ - muito elevado. Os pacientes sem risco (escore de 19 a 23) e de risco baixo (escore de 15 a 18) deverão ser avaliados a cada 72 horas e aqueles com graus de moderado e alto (escore $\leq 14$ ), a cada 24 horas. Utilizou-se o escore $\leq 16$ como crítico para o desenvolvimento de UP.

$\mathrm{Na}$ segunda etapa, para coletar informações relativas às condições preventivas, aos diagnósticos de Enfermagem, aos fatores de risco e à prescrição dos cuidados de Enfermagem, utilizou-se um formulário estruturado de prevenção de UP, o qual foi respondido com o auxílio do prontuário do paciente e dos dados colhidos por meio de exame físico diário da pele, realizado pelos pesquisadores.

As variáveis incluíram: sexo, faixa etária, cor, risco conforme a EB, classificação segundo o índice de massa corporal (IMC), presença de UP e comorbidades. Os resultados obtidos foram organizados em planilhas do Microsoft Excel 2007 e exportados para o programa estatístico IBM SPSS Statistics $20^{\circ}$. Os dados foram tratados segundo o teste bivariado de Pearson $\left(\chi^{2}\right)$, que estabelece testes estatísticos indicando o grau de associação entre variáveis intervenientes e de desfecho, considerando um nível de significância de $\mathrm{p} \leq 0,005$. Posteriormente, as informações foram tabuladas e apresentadas na forma de tabelas e gráficos, com suas respectivas distribuições percentuais.

\section{RESULTADOS}

Em relação ao aspecto sociodemográfico da amostra pesquisada, dos 20 pacientes analisados, 70\% eram do sexo feminino e 30\% do masculino. Quanto à faixa etária, $90 \%$ tinham idades entre 65 e 92 anos, o que caracteriza uma população idosa, seguido de $10 \%$ compreendidos na faixa etária de 20 a 32 anos. Observou-se que 60\% eram brancos; $7 \%$, pardos e $1 \%$, negros.

Ao analisar o escore total pela EB, $95 \%$ dos participantes apresentaram-no entre seis e nove, correspondendo assim a um risco muito elevado para desenvolver UP e 5\%, entre 16 e 17 com classificação de risco leve, demonstrando uma população com risco de surgimento de UP, como na Figura 1. Com relação à compleição física, $10 \%$ dos pacientes estavam com IMC normal, $25 \%$ estavam abaixo do peso, $15 \%$ acima do peso e $50 \%$ obesos. Pelas comorbidades analisadas nos pacientes da amostra, $35 \%$ dos participantes tinham diagnóstico médico de hipertensão arterial sistêmica (HAS), $20 \%$ de diabetes mellitus (DM) e $45 \%$ tinham ambos os diagnósticos, HAS e DM.

Observou-se também que não se utilizava nenhuma escala para predição de risco no setor, bem como nenhuma ferramenta que sinalizasse ao enfermeiro o risco que o paciente apresentava em relação ao surgimento de UP.

A Figura 2 mostra que, na maioria dos prontuários dos pacientes analisados, havia ausência dos registros de Enfermagem a respeito dos cuidados preventivos relacionados às UP, assim como a ausência da SAE. Com relação a estes, foram encontrados em um prontuário três prescrições de cuidados, realizadas por outro profissional e não pelo enfermeiro.

As ausências dessas medidas preventivas escritas no prontuário corroboravam com a clínica, uma vez que, ao ser feito o exame físico da pele diariamente, não se observavam quaisquer tipos de coberturas ou dispositivos que amenizassem os riscos no surgimento dessas lesões.

Nota-se, na Figura 3, o intervalo de dias entre a admissão e o surgimento de UP. Um total de $95 \%$ dos pacientes apresentou UP nos intervalos de quatro a oito dias de internação na UTI. Destes, 85\% tiveram UP estágio I; 5\%, UP

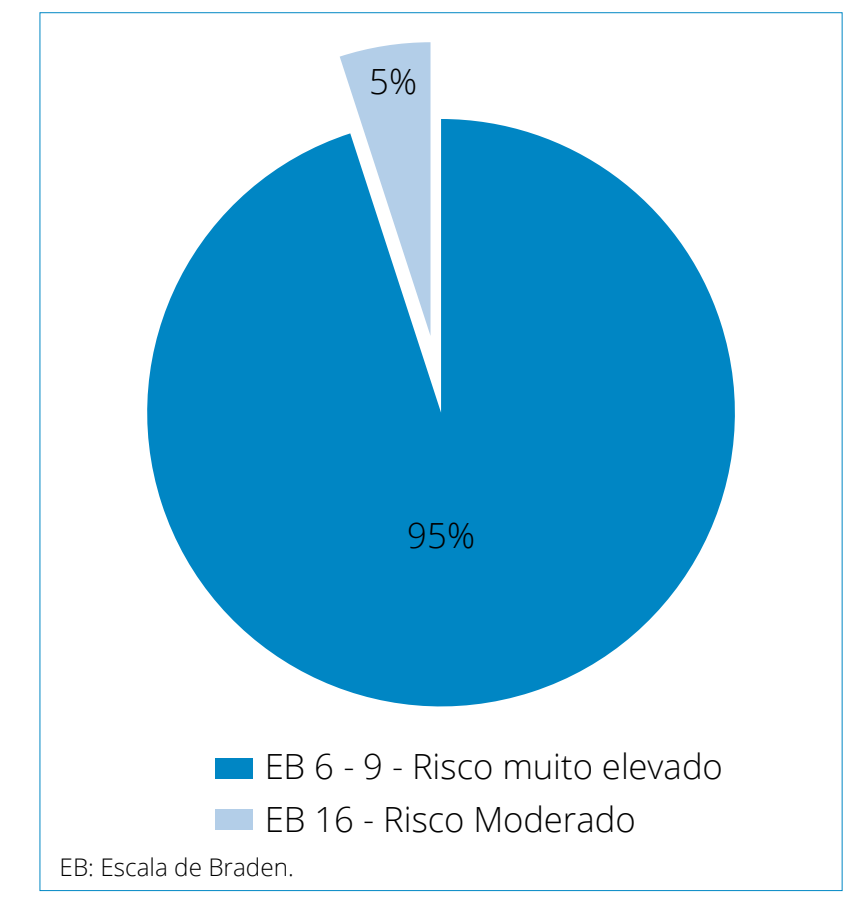

Figura 1. Classificação de risco segundo os escores da Escala de Braden dos pacientes na Unidade de Terapia Intensiva (Caruaru, 2014). 
por força de fricção ou cisalhamento em função das dobras dos lençóis e 5\% desenvolveram UP pela fixação inadequada da sonda vesical de demora.

$\mathrm{Na}$ associação das variáveis sociodemográficas e clínicas, observou-se diferença estatisticamente significativa para as variáveis faixa etária $(\mathrm{p}=0,002)$, risco pelo escore na $\mathrm{EB}$ $(\mathrm{p}=0,000)$, classificação do IMC $(\mathrm{p}=0,000)$, surgimento de UP $(p=0,000)$ e presença de comorbidades $(p=0,000)$, conforme demonstrado na Tabela 1 .

\section{DISCUSSÃO}

Os pacientes que necessitam de internação em UTI, em geral, precisam de um longo tempo de permanência nela e de cuidados contínuos. Estudos na literatura demonstram que a incidência de UP em pacientes hospitalizados em UTI, no Brasil, varia de 10,6 a 55\%14-17.

Com relação ao sexo, do total de pacientes com UP, houve uma população significativamente feminina (70\%). Em um estudo que explica a predominância de UP no sexo feminino, explicou-se que as mulheres apresentam maior longevidade do que os homens, levando-as a tempos mais longos de doenças crônicas, além de geralmente as mulheres procurarem mais os serviços de saúde do que os homens, o que pode explicar o maior percentual do sexo feminino ${ }^{10}$.

$\mathrm{O}$ cruzamento entre o surgimento de UP e sexo apresentou-se mais frequente no grupo estudado do sexo feminino (70\%). Estudos apontam divergências em relação a este contexto, alguns com predominância de risco no sexo feminino ${ }^{16-18}$ e outros no masculino ${ }^{19-21}$.

Entre algumas doenças crônicas não transmissíveis, o DM e a HAS podem alterar a circulação sanguínea, a oxigenação, as alterações dos níveis de eletrólitos e proteínas e o nível perceptivo e de consciência; desse modo, prejudicando

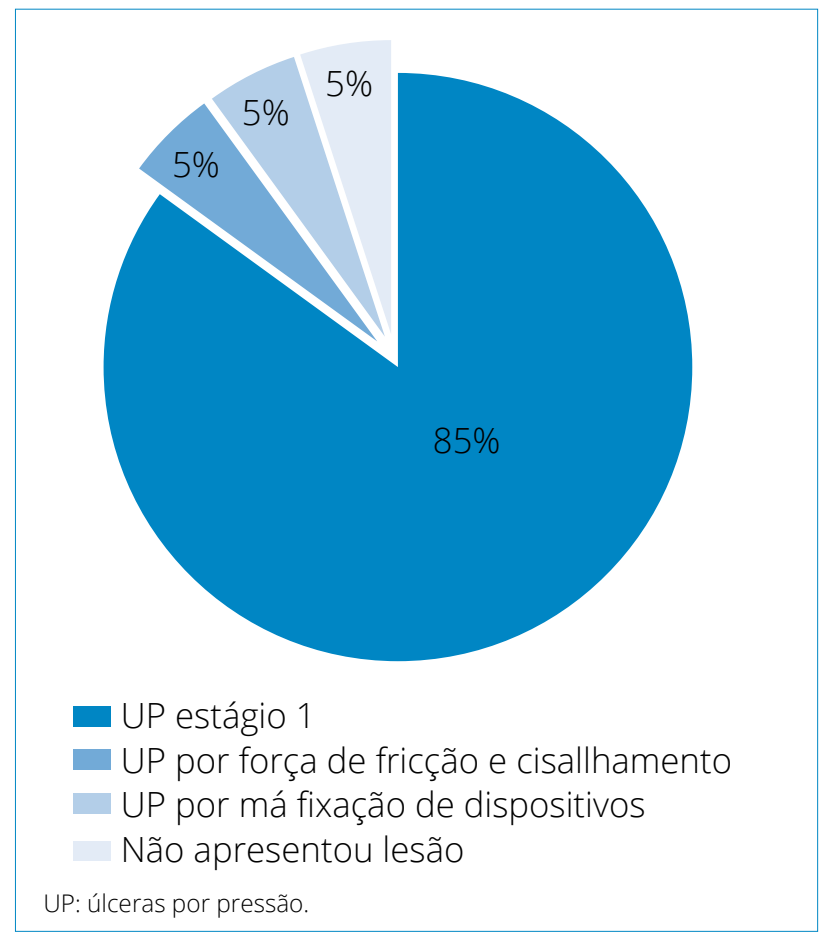

Figura 3. Úlceras por pressão presentes no intervalo de quatro a oito dias (Caruaru, 2014).

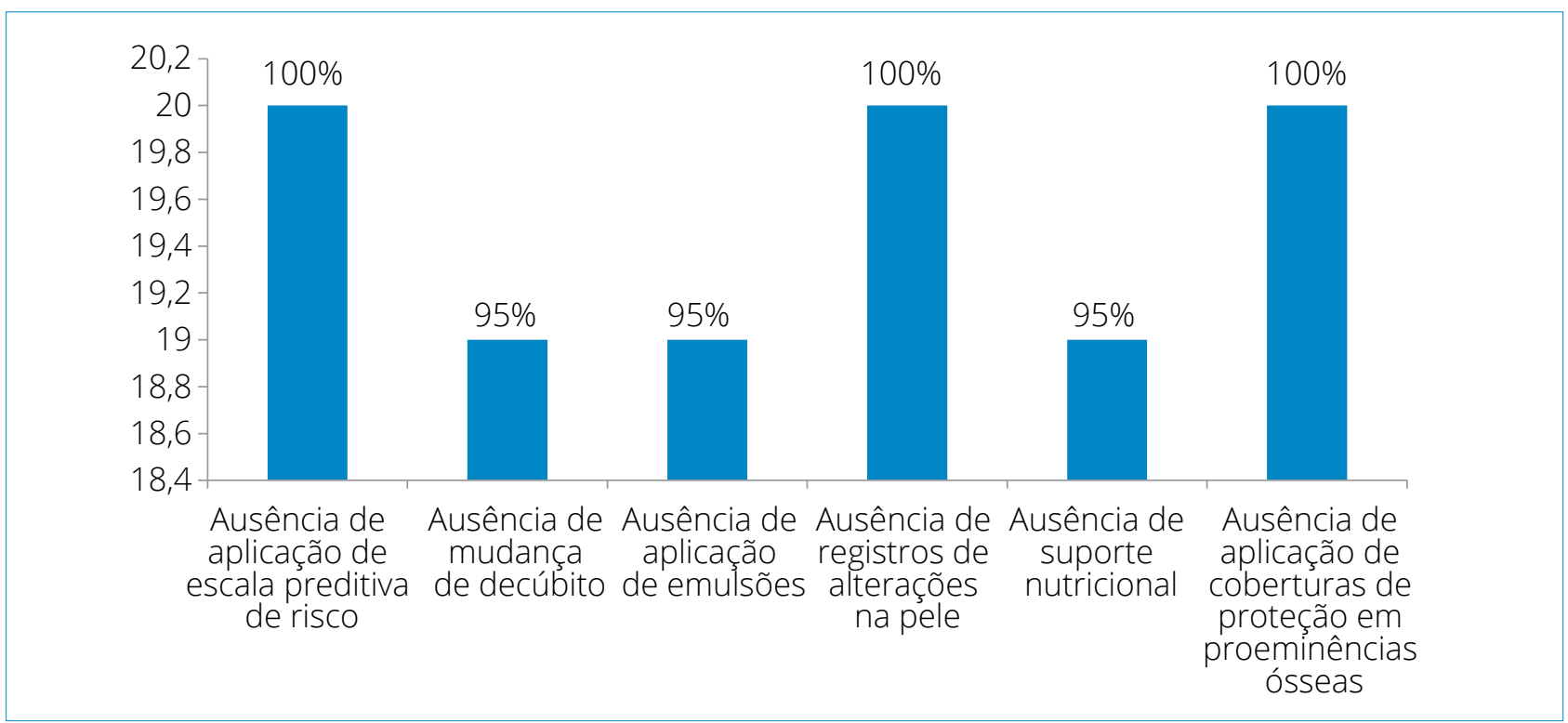

Figura 2. Cuidados de Enfermagem não implementados para a prevenção de úlcera por pressão na Unidade de Terapia Intensiva (Caruaru, 2014). 
Tabela 1. Associação entre a ausência dos cuidados de Enfermagem e as variáveis.

\begin{tabular}{|c|c|c|c|c|c|c|c|}
\hline \multirow{3}{*}{ Variáveis } & \multicolumn{4}{|c|}{ Ausência dos cuidados de Enfermagem } & \multirow{2}{*}{\multicolumn{2}{|c|}{ Total }} & \multirow{3}{*}{ Valor $\mathrm{p}$} \\
\hline & \multicolumn{2}{|c|}{ Sim } & \multicolumn{2}{|c|}{ Não } & & & \\
\hline & $\mathrm{n}$ & $\%$ & $\mathrm{n}$ & $\%$ & $\mathrm{n}$ & $\%$ & \\
\hline \multicolumn{8}{|l|}{ Sexo } \\
\hline Masculino & 5 & 23,3 & 1 & 100 & 6 & 100 & \multirow{2}{*}{0,117} \\
\hline Feminino & 14 & 73,7 & 0 & 0 & 14 & 100 & \\
\hline \multicolumn{8}{|l|}{ Faixa etária } \\
\hline 20 a 32 anos & 1 & 5,3 & 1 & 5,3 & 2 & 100 & \multirow{2}{*}{0,002} \\
\hline 65 a 92 anos & 18 & 94,7 & 0 & & 18 & 100 & \\
\hline \multicolumn{8}{|l|}{ Cor } \\
\hline Branca & 12 & 63,2 & 0 & 0 & 12 & 100 & \multirow{3}{*}{0,376} \\
\hline Parda & 6 & 31,6 & 1 & 14,3 & 7 & 100 & \\
\hline Negra & 1 & 5,3 & 0 & 0 & 1 & 100 & \\
\hline \multicolumn{8}{|l|}{ Risco da Escala Braden } \\
\hline Moderado & 0 & 0 & 1 & 5 & 1 & 100 & \multirow{2}{*}{0,000} \\
\hline Muito elevado & 19 & 95 & 0 & 0 & 19 & 100 & \\
\hline \multicolumn{8}{|c|}{ Classificação conforme índice de massa corporal } \\
\hline Eutrófico & 0 & 0 & 1 & 5 & 1 & 100 & \multirow{2}{*}{0,000} \\
\hline Distrófico & 19 & 95 & & & 19 & 100 & \\
\hline Presença de úlcera por pressão & 19 & 95 & 1 & 5 & 20 & 100 & 0,000 \\
\hline Presença de comorbidades & 19 & 95 & 1 & 5 & 20 & 100 & 0,000 \\
\hline
\end{tabular}

Nota: $p \leq 0,005$ - teste do $\chi^{2}$.

a cicatrização da pele e reduzindo a sua resistência às lesões pela fragilidade advinda das referidas alterações ${ }^{22}$. No presente estudo, todos os pacientes eram portadores de ao menos um destes diagnósticos e, dentre eles, $45 \%$ tinham ambas, DM e HAS.

Um dos fatores relevantes para o desenvolvimento de UP é a idade. Estudos em literaturas nacionais e internacionais indicam que esse provável aumento para o risco de UP acontece em função das alterações específicas no processo de envelhecimento da pele, pois resultam num tecido mais frágil e suscetível às forças mecânicas, tais como fricção, pressão e cisalhamento ${ }^{23}$. Assim, $90 \%$ dos pacientes do estudo tinham entre 65 e 92 anos, o que indica que esta população seja mais propícia ao desenvolvimento de UP. Os cruzamentos estatísticos entre a ausência das ações de Enfermagem nesta faixa etária apresentaram-se significativos $(p=0,002)$, bem como a análise realizada entre faixa etária e surgimento de UP ( $\mathrm{p}=0,003)$.

A diminuição do nível de consciência em decorrência das doenças de origem neurológica ou da percepção sensorial reduzem a mobilidade e a atividade do paciente, dificultando-o de perceber desconforto e/ou dor, o que ocasiona o desenvolvimento de UP. Dentre as modificações da mobilidade e neurológicas, destacam-se coma, imobilização e parestesias ${ }^{24}$. Observou-se, no presente estudo, na subescala percepção sensorial, que $90 \%$ dos pacientes apresentavam-se completamente limitados, enquanto que, na mobilidade, 95\% dos sujeitos estavam imóveis.

Uma grande parte dos pacientes estudados, $90 \%$, apresentou a pele raramente úmida, ou seja, frequentemente seca, além de gerar um menor desgaste físico para a equipe de Enfermagem, pois o paciente somente era trocado nos momentos rotineiros, minimizando o risco de desenvolver UP. A umidade pode estar presente pelas alterações no nível de consciência e outras complicações no sistema neurológico periférico. Dentre estas, destacam-se a transpiração excessiva e as incontinências urinária e fecal. As drenagens de feridas, as secreções de drenos e os restos alimentares também são outros fatores que favorecem a exposição do paciente à umidade. Assim, quanto ela está presente durante um tempo prolongado, pode desencadear a maceração e a ruptura da pele, que são, consequentemente, um fator determinante para o surgimento da UP ${ }^{24}$.

$\mathrm{O}$ aspecto nutricional representa um fator determinante para o surgimento de tais lesões; portanto, $95 \%$ dos pacientes tinham como item a nutrição provavelmente inadequada composta na $\mathrm{EB}$ e analisada por meio do formulário estruturado da pesquisa. Neste contexto, a 
avaliação do estado nutricional torna-se relevante, pois determinará a conduta terapêutica nutricional a fim de manter o organismo com um adequado aporte nutricional, facilitando a sua capacidade de manter a integridade tissular da pele, além de favorecer a regeneração e o processo cicatricial da pele ${ }^{25}$.

Desse modo, dentre os pacientes com risco elevado ao desenvolvimento de UP (95\%), segundo a EB entre seis e nove, observou-se que $50 \%$ apresentaram IMC $\geq 38$, classificados assim como obesos. Isto denota que, além da sinalização pela $\mathrm{EB}$, o paciente apresenta outro risco para o desenvolvimento dessas lesões, necessitando de cuidados específicos voltados a essa população. A análise estatística dos pacientes que indicaram distrofia, ou seja, tinham algum tipo de irregularidade no IMC, aponta significância estatística para os achados $(\mathrm{p}=0,000)$ quando comparados com a ausência dos cuidados de Enfermagem.

Com relação à força de fricção e cisalhamento, $85 \%$ dos sujeitos mostraram sinais de problema, isto é, dependiam de auxílio para se mover no leito. Este item avalia o grau de atrito entre a pele e o lençol do paciente. A fricção é gerada quando as forças dessas duas superfícies deslizam uma contra a outra, o que resulta em bolhas ou queimaduras. $\mathrm{O}$ cisalhamento é uma consequência de mobilização ou posicionamento incorretos, ocasionando danos mais profundos ${ }^{26}$.

Frente ao surgimento de UP, analisando o intervalo de dias, observou-se uma média de 6 dias $(\sigma=1,47)$ sendo o tempo mínimo de quatro e o máximo de seis dias. Este dado corrobora com a evidência do período médio de surgimento de UP em pacientes críticos, que se caracteriza acima de 72 horas desde a sua admissão ${ }^{22,23}$.

Diante dos riscos no desenvolvimento de UP, foram notados escores na $\mathrm{EB}$, os quais indicaram que $95 \%$ dos pacientes apresentaram risco muito elevado. Após este dado, com o risco definido, o profissional enfermeiro, por meio da SAE, deverá elaborar medidas, considerando as limitações e possibilidades individuais, objetivando minimizar os riscos do paciente para desenvolver UP ${ }^{24}$.

Neste contexto, a taxonomia II da NANDA I define o seguinte diagnóstico relacionado ao comprometimento tissular: risco de integridade da pele prejudicada. Levando-se em conta o capítulo das ligações NIC-NANDA-I, encontram-se as intervenções prioritárias: prevenção de UP, controle da pressão, supervisão da pele, cuidados da pele, tratamentos tópicos, monitorização de sinais vitais, cuidados na incontinência urinária, posicionamento e terapia nutricional ${ }^{13}$.
Neste estudo, por meio do cruzamento de dados, dos pacientes que desenvolveram UP, 95\% tiveram ausência das ações preventivas de Enfermagem, com significância estatística ( $\mathrm{p}=0,000)$, sendo que tal dado considera ainda a presença de UP como uma iatrogenia por parte da equipe ${ }^{27}$. Portanto, o enfermeiro possui ações determinantes na prevenção de UP, uma vez que este apresenta risco de desenvolver lesão segundo um instrumento preditivo, o qual, no caso deste estudo, é a EB. As rotinas de prevenção incluem ${ }^{28}$ :

- avaliar o grau de risco, numa assistência individualizada, como uma confecção de um protocolo para prevenção de UP;

- empregar as escalas de avaliação do grau de risco, como a EB adaptada para a língua portuguesa;

- providenciar colchão de poliuretano (colchão caixa de ovo) para o paciente;

- identificar os fatores de risco e direcionar o tratamento preventivo, modificando os cuidados conforme os fatores individuais;

- mobilizar ou mudar o paciente de posição de duas em duas horas, bem como realizar massagem de conforto com emulsão;

- proteger as saliências ósseas, principalmente calcâneas, com rolos e travesseiros;

- registrar alterações da pele do paciente seguindo os estágios de classificação das UP, propostos pelo National Pressure Ulcer Advisory Panel (NPUAP);

- realizar tratamento precoce da pele, ou seja, manter e melhorar a tolerância tissular à pressão, a fim de prevenir lesão;

- checar áreas vulneráveis da pele de todos os pacientes em risco e otimizar o estado dessa pele, por meio da hidratação com cremes à base de ácidos graxos essenciais, tratar a incontinência, evitar o uso de água muito quente e providenciar suporte nutricional;

- monitorar e documentar as intervenções e os resultados obtidos;

- implementar medidas de suporte mecânico, ou seja, proteger/evitar complicações adversas de forças mecânicas externas;

- criar e fornecer um programa de ensino aos pacientes de risco em longo prazo para as pessoas que cuidam deles.

Desse modo, o enfermeiro, por sua cientificidade, é o profissional capaz de desenvolver ações de intervenção a fim de minimizar os riscos no surgimento de tais lesões, o que foi percebido como ausente neste estudo, demonstrando um déficit no cuidado voltado a estes pacientes e, consequentemente, o desenvolvimento de UP. 


\section{CONCLUSÃO}

Os resultados obtidos neste estudo demonstram que houve um déficit das ações de Enfermagem voltadas à prevenção de UP, desde a SAE às práticas assistenciais do cuidado, consequentemente, o surgimento das úlceras. Não se utilizava a EB como ferramenta de predição de risco, bem como não foram observados registros nos prontuários que apontassem medidas preventivas para diminuírem os riscos no surgimento de tais lesões.

O intervalo de dias para o aparecimento de UP na UTI foi em média de seis dias $(\sigma=1,47)$, observados a partir de quatro dias da admissão do paciente. A ausência de ações preventivas de Enfermagem contribuiu para o desenvolvimento de UP ( $\mathrm{p}=0,000)$.

Assim, torna-se relevante a implementação de uma escala de avaliação de risco e de protocolos de prevenção dessas lesões a fim de fornecer subsídios ao profissional enfermeiro na prestação de uma assistência de qualidade e individualizada.

O enfermeiro é o membro da equipe de Enfermagem respaldado técnico-cientificamente para instituir ações que visem minimizar os eventos adversos a que o paciente esteja exposto. Contudo, o mesmo torna-se responsável por adotar medidas que busquem reduzir o surgimento de UP. Porém, tais medidas estavam ausentes neste estudo.

Nesse sentido, a EB configura-se como um instrumento de fácil utilização, que demonstra a probabilidade, de forma individual, que o paciente apresenta em desenvolver uma UP. Por meio do escore da EB, o enfermeiro terá subsídios para desenvolver de forma sistemática tais ações, e a ausência delas poderá caracterizar negligência por parte dos profissionais, tendo em vista que a $\mathrm{EB}$, por si só, demonstra que o paciente possui uma probabilidade de ter tal lesão.

\section{REFERÊNCIAS}

1. Rogenski NMB, Kurcgant P. The incidence of pressure ulcers after the implementation of a prevention protocol. Rev Latino-Am Enfermagem [Internet]. 2012 [cited 2015 Apr 22]; 20(2):333-9. Available from: http://www.scielo.br/scielo. php?script=sci_arttext\&pid=S010411692012000200016\&ln $\mathrm{g}=\mathrm{en} \& \mathrm{nrm}=\mathrm{iso}$

2. Alves AG, Borges JW, Brito MA. Avaliação do risco para úlcera por pressão em unidades de terapia intensiva: uma revisão integrativa. J Res Fundam Care [Internet]. 2014 [cited 2015 Apr 28];6(2):793-804. Available from: http://www.seer.unirio.br/ index.php/cuidadofundamental/article/view/3004/pdf_1287

3. Brasil. Portaria MS/GM 529, de 1 de abril de 2013. Institui - Programa Nacional de Segurança do Paciente (PNSP). Brasília; 2013.

4. Freire IM. Assistência de enfermagem a portador de úlceras por pressão, tratado com hidrofibra com prata iônica. Porto Alegre: Congresso Brasileiro de Estomaterapia; 2011.

5. Lima AC, Guerra DM. Avaliação do custo do tratamento de úlceras por pressão em pessoas hospitalizados usando curativos industrializados. Ciênc Saúde Coletiva [Internet]. 2011 [cited 2015 Jan 28];6(1):267-77. Available from: http:// www.scielo.br/scielo.php?script=sci_arttext\&pid=S141381232011000100029\&lng=en\&nrm=iso

6. Silva DP, Barbosa MH, Araujo DF, Oliveira LP, Melo AF. Úlcera por pressão: avaliação de fatores de risco em pacientes internados em um hospital universitário. Rev Eletrônica Enferm [Internet]. 2011 [cited 2014 Dec 14];13(1):118-23. Available from: http:// www.fen.ufg.br/revista/v13/n1/v13n1a13.htm

7. Malagutti W, Kakihara CT. Curativo, ostomias e dermatologia: uma abordagem multiprofissional. São Paulo: Martinari; 2010.

8. Almeida RA, Bastos RA, Almeida FC, Pequeno GA, Vasconcelos JM, Rodrigues FA. Avaliação da utilização de protocolos na prevenção de úlceras por pressão. Rev Cienc Saúde [Internet]. 2012 [cited 2014 Dec 14];5(2):125-31.
Available from: http://revistaseletronicas.pucrs.br/ojs/index. php/faenfi/article/view/10457/8207

9. Braden BJ, Bergstrom N. Clinical utility of Braden Scale for predicting pressure sores risk. Decubitus [Internet]. 1989 [cited 2014 Dec 14];2(3):44-51. Available from: http:// journals.Iww.com/aswcjournal/Citation/1989/08000/ CLINICAL_UTILITY_OF_THE_BRADEN_SCALE_FOR.9.aspx

10. Paranhos WY, Santos VL. Avaliação de risco para úlceras por pressão por meio da Escala de Braden, na língua portuguesa. Rev Esc Enferm USP [Internet]. 1999 [cited 2015 Jan 22];33(n.esp.):191-206. Available from: http://www. ee.usp.br/reeusp/upload/pdf/799.pdf

11. Gonçalves LR, Nery IS, Nogueira LT, Bonfim EG. O desafio de implantar a sistematização da assistência de enfermagem sob a ótica do discente. Esc Anna Nery [Internet]. 2007 [cited 2015 Jan 08];11(3):459-65. Available from: http://www. scielo.br/pdf/ean/v11n3/v11n3a10.pdf

12. Carvalho EC, Bachion MM. Processo de enfermagem e sistematização da assistência de enfermagem - intenção de uso por profissionais de enfermagem. Rev Eletr Enferm [Internet]. 2009 [cited 2015 Feb 05];11(3):466. Available from: http://www.fen.ufg.br/revista/v11/n3/pdf/v11n3a01.pdf

13. Baravesco T, Lucena AF. Intervenções da classificação de enfermagem NIC validadas para pacientes em risco de úlcera por pressão. Rev Latino-Am. Enferm [Internet]. 2012 [cited 2015 May 26]. Available from: http://dx.doi.org/10.1590/ S0104-11692012000600013

14. Albuquerque AM, Souza MA, Torres VS, Porto VA, Soares MJ, Torquato IM. Avaliação e prevenção de Úlcera por Pressão pelos enfermeiros de Terapia Intensiva: Conhecimento e Prática. Rev Enferm UFPE [Internet]. 2014 [cited 2015 Apr 30];8(2):229-39. Available from: http://www.revista.ufpe.br/ revistaenfermagem/index.php/revista/article/view/4688/ pdf_450 
15. Rodrigues MM, Souza MS, Silva JL. Sistematização da Assistência de Enfermagem na prevenção da lesão tecidual por pressão. Cogitare Enferm [Internet]. 2008 [cited 2014 July 12];13(4):566-75. Available from: http://ojs.c3sl.ufpr.br/ojs2/index.php/cogita re/article/ view/13117/8875

16. Fernandes NC, Torres GV. Incidência e fatores de risco de úlceras de pressão em pacientes de unidade de terapia intensiva. Cienc Cuid Saúde. 2008;7(3):304-10.

17. Fernandes LM, Caliri MH. Uso da escala de Braden e de Glasgow para identificação do risco para úlceras de pressão em pacientes internados em centro de terapia intensiva. Rev Latino-Am Enferm. 2008;16(6):973-8.

18. Araújo CR, Lucena ST, Santos IB, Soares MJ. A enfermagem e a utilização da escala de Braden em úlcera por pressão. Rev Enferm UERJ. 2010;18(3):359-64.

19. Fernandes MG, Costa KN, Santos SR, Pereira MA, Oliveira DS, Brito SS. Risco para úlcera por pressão em idosos hospitalizados: aplicação da escala de Waterlow. Rev Enferm. UERJ. 2012;20(1):56-60.

20. Araújo TM, Araújo MF, Cavalcante CS, Barbosa Júnior GM, Caetano JA. Acurácia de duas escalas de avaliação de risco para úlcera por pressão em pacientes críticos. Rev Enferm UERJ. 2011;19(3):381-5.

21. Studart RM, Melo EM, Lopes MV, Barbosa IV, Carvalho ZM. Tecnologia de enfermagem na prevenção da úlcera por pressão em pessoas com lesão medular. Rev Bras Enferm. 2011;64(3):494-500.

22. Bavaresco T, Medeiros RH, Lucena AF. Implantação da Escala de Braden em uma unidade de terapia intensiva de um hospital universitário. Rev Gaúcha Enferm. 2011;32(4)703-10.
23. Freitas MC, Medeiros AB, Guedes MV, Almeida PC, Galiza FT, Nogueira JM. Úlcera por pressão em idosos institucionalizados: análise da prevalência e fatores de risco. Rev Gaúcha Enferm [Internet]. 2011 [cited 2015 May 12]. Available from: http://www.scielo.br/scielo.php?pid=S198314472011000100019\&script=sci_arttext

24. Rogenski NM, Kurcgant P. Avaliação da concordância na aplicação da Escala de Braden inter observadores. Acta Paulista Enferm [Internet]. 2012 [cited 2015 May 18]. Available from: http://www.unifesp.br/acta/artigo.php?volu me=25\&ano=2012\&numero=1\&item

25. Nogueira GA, Assad LG. Avaliação de risco para úlcera por pressão: contribuição para o cuidado de enfermagem na unidade de clínica médica. Rev Enferm UFPE [Internet]. 2013 [cited 2015 May 22];7(11):6462-70. Available from: http:// www.revista.ufpe.br/revistaenfermagem/index.php/revista/ article/download/4766/7743

26. Serpa LF, Santos VL, Campanili TC, Queiroz M. Predictive validity of the Braden scale for pressure ulcer risk in critical care patients. Rev Latino-Am Enferm [Internet]. 2011 [cited 2015 May 01];19(1):50-7. Available from: http://www.ncbi. nlm.nih.gov/pmc/articles/PMC4042540/

27. Sila EW, Araújo RA, Oliveira EC, Falcão VT. Aplicabilidade do protocolo de prevenção de úlcera de pressão em unidade de terapia intensiva. Rev Bras Ter Intensiva [Internet]. 2010 [cited 2015 Apr 18];22(2):175-85. Available from: http://www. scielo.br/pdf/rbti/v22n2/a12v22n2.pdf

28. Medeiros $A B$, Lopes $C H$, Jorge MS. Análise da prevenção e tratamento das úlceras por pressão propostos por enfermeiros. Rev Esc Enferm USP [Internet]. 2009 [cited 2015 Apr 20];43(1): 223-8. Available from: http://www.scielo. $\mathrm{br} / \mathrm{pdf} /$ reeusp/v43n1/29.pdf 